\title{
INDUSTRIA FORESTAL Y MERCADO DE LA MADERA: NAVEGANDO EN LA TORMENTA
}

\author{
Juan Picos Martín
}

Universidade de Vigo. Dpto. Enxeñería dos Recursos Naturais e Medio Ambiente. Escola de Enxeñería Forestal. Campus A Xunqueira s/n. 36005-PONTEVEDRA (España). Correo electronico: jpicos@uvigo.es

Behold the threaden sails, Borne with the invisible and creeping wind, Draw the huge bottomes through the furrow'd sea,

Breasting the lofty surge.

William Shakespeare, Henry V Act III.

\section{Resumen}

El artículo se centra sobre la situación en la que se encuentra la industria forestal/madera en el contexto económico actual. En la primera parte se relata la evolución en la última década a escala global, europea y española, con algunas referencias a Galicia. En la segunda parte se apunta cuales serán las claves para el sector en el futuro inmmediato

Palabras Clave: Crisis, Recesión, Recuperación, Economía, España

\section{INTRODUCCIÓN}

En Diciembre de 2006, Paul Krugman, a la postre Premio Nobel de Economía 2008, escribía un artículo de opinión titulado "Signos de tormenta económica" (KRUGMAN, 2006) en el que advertía del inminente periodo recesivo que se avecinaba inminentemente sobre la economía norteamericana y por extensión sobre la economía global. Desde entonces han sido numerosas las parábolas sobre la situación económica que han empleado el símil de la tormenta.

Una parte importante de mi infancia y juventud estuvo presidida, desde la pared del salón familiar, por una foto del Galatea, Buque Escuela de la Armada Española, de tan inmaculado servicio como lamentable final. Durante la etapa en la que el Galatea fue buque escuela de maniobra para aprendices de marineros especialistas navegó mi abuelo Juan. Por ello cuando la organización del $6^{\circ}$ Congreso Forestal Español me invitó amablemente a dar una ponencia sobre el mercado de la madera y la industria forestal, no pude por menos que recuperar la metáfora de Krugman y pensar en un sector bamboleado como un velero en la galerna.

\section{LA INDUSTRIA FORESTAL: NAVEGANTE EN UN MUNDO EN CAMBIO}

En la UNECE (formada por los 56 estados de América del Norte, Europa y la llamada Comunidad de Estados Independientes -CISque actualmente ocupan el territorio que fue la URSS) cerca de 300.000 millones US\$ de actividad económica depende de la producción de materias primas procedentes de la gestión de masas forestales. Las actividades económicas del "sector forestal" incluyendo selvicultura, 
gestión forestal, industria de la Madera e industria de la pasta y el papel suponen el $1 \%$ del PIB en Europa y América del Norte y el 0,8\% para Rusia. El Valor añadido por el sector forestal de la UNECE es de 285.000 millones US\$, del que el más del $80 \%$ corresponde a las industrias de la Madera y el papel. En algunos países la contribución al PIB del sector forestal es netamente superior a la media. Este es el caso de países como Finlandia $(5,7 \%)$, Suecia $(3,8 \%)$, Estonia $(3,7 \%)$, Letonia $(3,4 \%)$, Canadá $(2,7 \%)$, Bosnia y Herzegovina (2,5\%), Lituania $(2,4 \%)$, Austria, Bielorrusia o República Checa $(2,1 \%)$.

El contexto que rodea a la Industria Forestal ha sufrido intensos cambios en las últimas décadas. Algunos de ellos son fruto de inexorables cambios que, poco a poco, han ido modelando nuevos escenarios para el comercio de la madera y los productos forestales a escala global. La caída del bloque comunista, la globalización económica y la emergencia de países asiáticos han alterado decisivamente los mercados. Pero no solo las tendencias económicas y políticas han influido, los cambios tecnológicos y sociales, sobre todo en los países desarrollados, han generado nuevas demandas y cambios en la distribución y circuitos comerciales que han obligado al sector a adaptarse.

En los primeros años del siglo XXI muchos de los países en vías de desarrollo han incrementado de manera radical su apoyo para implantación y desarrollo de industrias forestales. (FAO, 2011). Esta aparición y crecimiento de productores de bajo coste ha propiciado un cambio paulatino, pero difícilmente reversible, en los flujos de comercio mundial (Figuras 1, 2, 3).

Independientemente de su lugar de producción, actualmente, la mayor parte del comercio de productos forestales tiene lugar en Europa, en América del Norte y entre los países asiáticos, europeos y norteamericanos. Europa es la región exportadora e importadora más grande en el mundo, pero, en los últimos años, la Federación Rusa se ha erigido como el mayor exportador de madera en rollo industrial para abastecer a Asia Oriental y Europa. Por su parte, China ha llegado a ser el importador más importante de madera y un exportador e importador decisivo productos elaborados de madera y papel. América del Sur se ha erigido como el más dinámico polo industrial en el sector de la pasta y el

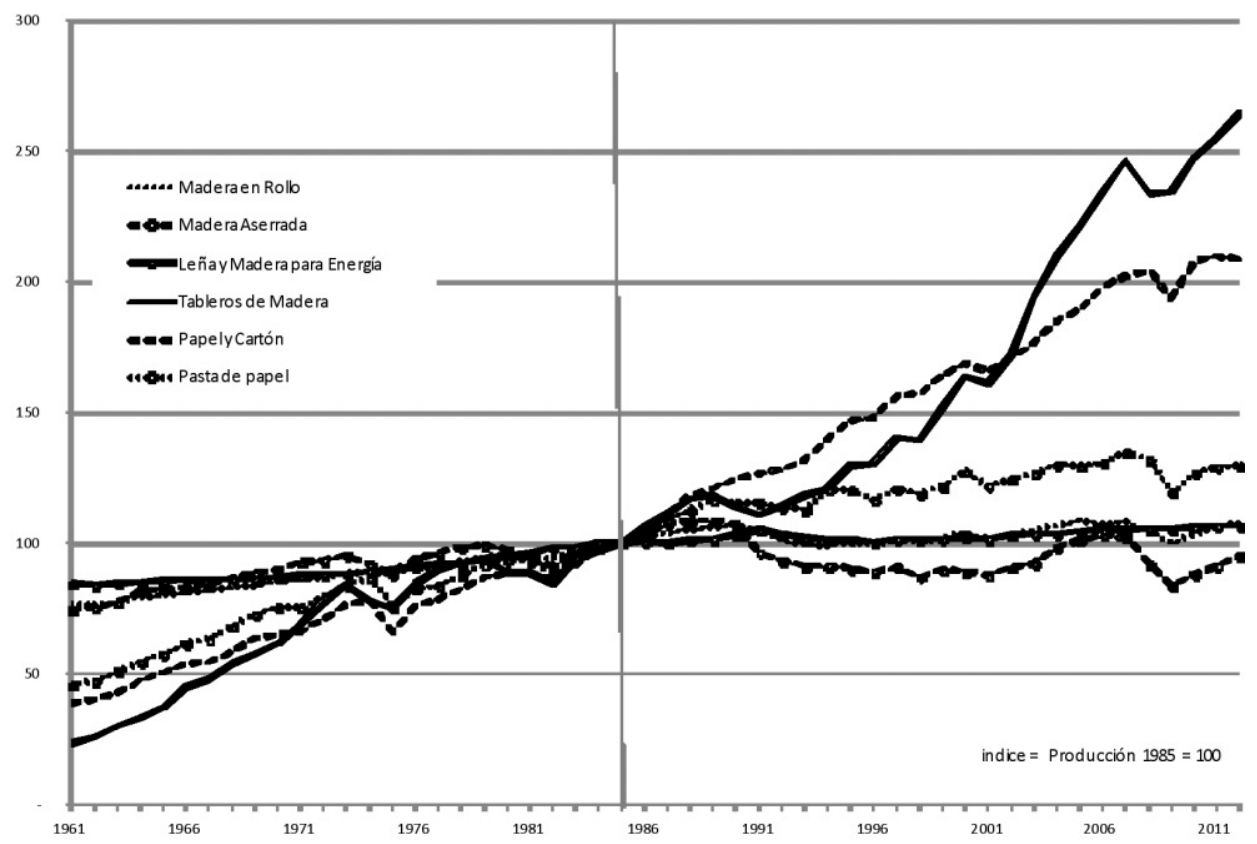

Figura 1. Evolución de la Producción Mundial 1961 - 2012 (Base 1985 = 100) 


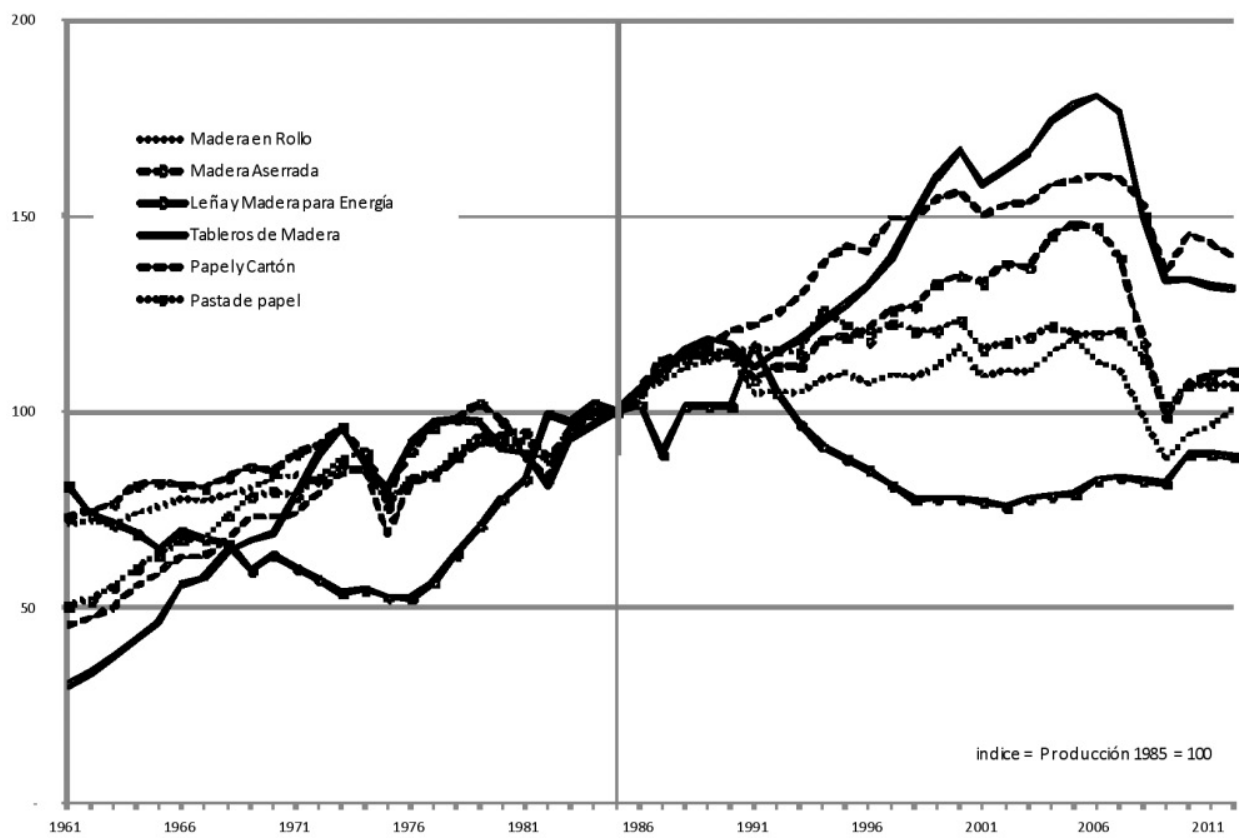

Figura 2. Evolución de Producción de A. del Norte y Europa (excepto E. del Este) 1961-2012 (Base 1985 = 100)

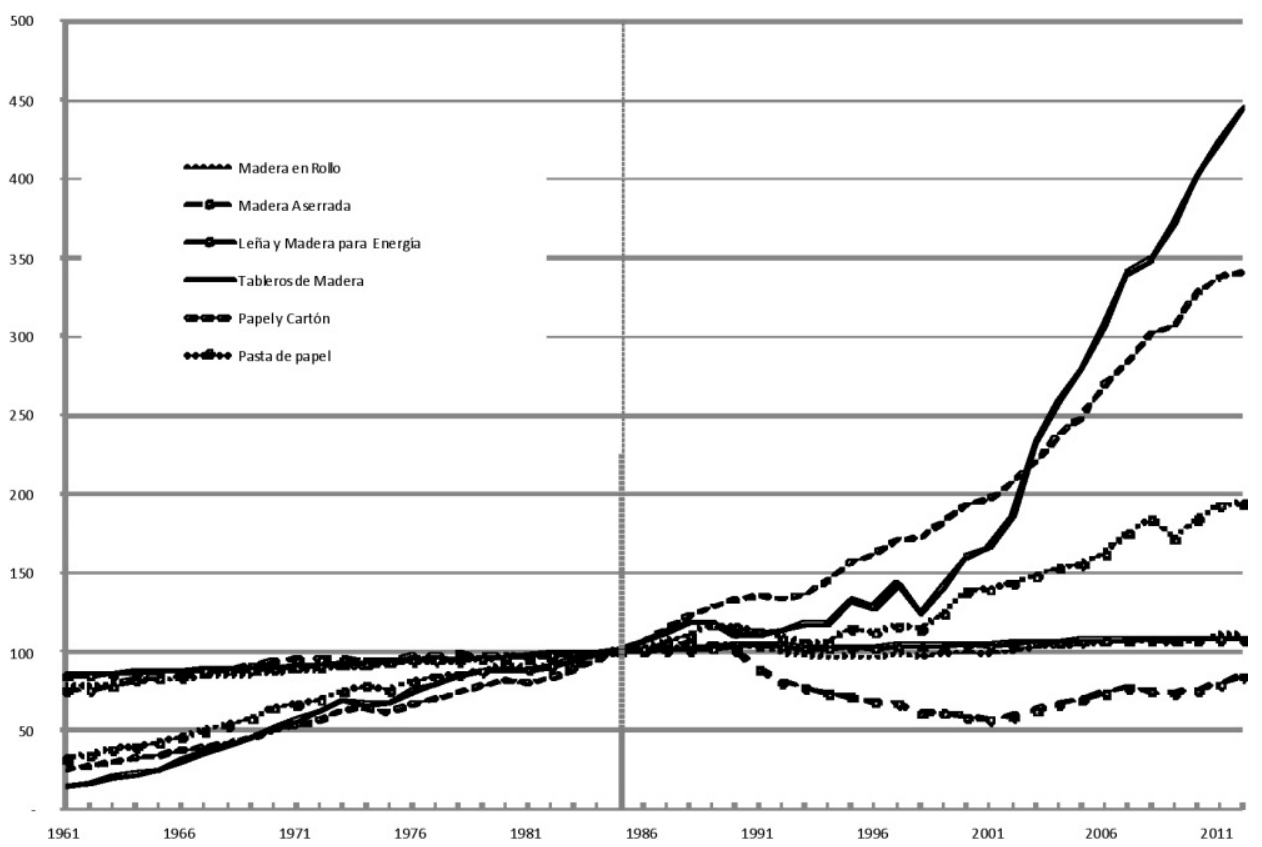

Figura 3. Evolución de Producción Mundial sin A. del Norte y Europa (e incl E. del Este) 1961-2012 (Base 1985 = 100) 


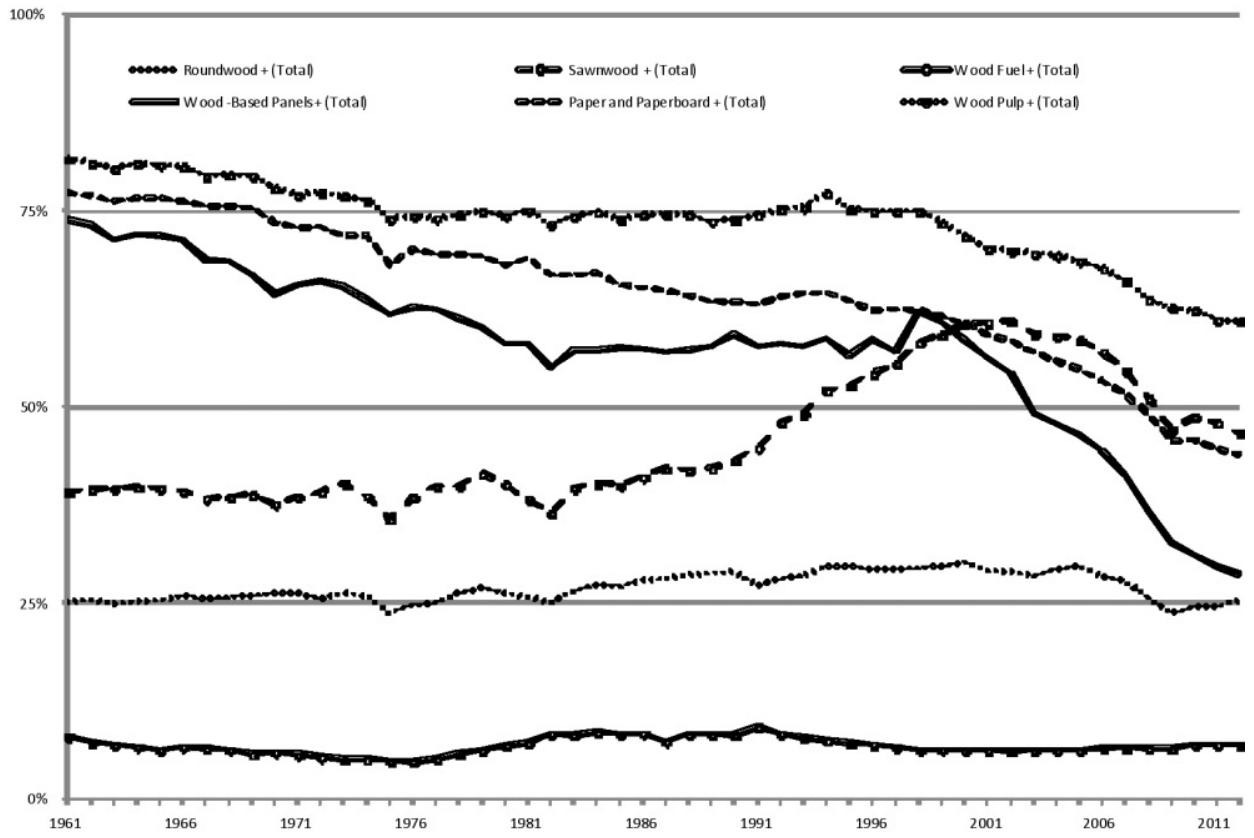

Figura 4. Cuota mundial de producción de América del Norte y Europa (excepto Europa del Este)

papel, especialmente aquel basado en fibras cortas (eucalipto). Las industrias de mobiliario y otras segundas transformaciones de madera están aumentando significativamente en varios países en desarrollo y en Europa oriental.

Simultáneamente conceptos como "gestión forestal sostenible", "certificación forestal", "responsabilidad social", "derechos de emisión", "mercados de carbono", "lucha contra comercio ilegal de madera" fueron impregnando el día a día del sector, condicionando políticas públicas y modificando también el statu quo del sector.

Mientras la industria forestal y los mercados de productos forestales, iban digiriendo estos nuevos condicionantes económicos, sociales y ambientales, la economía mundial se vio golpeada a partir de 2008 por una severa recesión de raíces muy profundas y complejas.

\section{LA GRAN RECESIÓN: LA TORMENTA PERFECTA}

El colapso del mercado inmobiliario fue el resultado de años de crédito fácil (hipotecas $s u b$ - prime), ausencia de disciplina crediticia, desarrollo de prácticas financieras perversas y excesiva construcción de viviendas. Todos estos factores llevaron a la contracción brutal del crédito. El resultado final fue un colapso financiero y económico en los Estados Unidos de América que se transformó en una crisis mundial de grandes proporciones. Así, la llamada Gran Recesión (ELSBY et al., 2010) se erigió como el más duradero periodo de crisis económica en tiempos de paz desde la Gran Depresión de los años 30.

Por si el panorama global no fuera suficiente grave, la Unión Europea (UE), y la Zona Euro (ZE) en particular, se encuentran en una situación muy delicada. Europa se enfrenta a limitaciones estructurales de su economía - la mayoría de las cuales se manifiestan en su limitada competitividad - y las actuales dificultades a que se enfrenta con relación a la sostenibilidad de las finanzas públicas y del sector financiero, en muchos Estados Miembros - entre ellos España - que afectan al conjunto de la ZE.

Las primeras muestras de recuperación se evidenciaron en algunos países en 2009 e incluso en países como EE.UU. la Gran Recesión ha sido 
dada "técnicamente" por superada (NBER, 2010). Sin embargo sus efectos continúan a afectar gravemente la economía mundial y aunque a partir de 2010 se ha producido un modesto crecimiento económico global, desde mediados de 2012, dicho crecimiento ha comenzado a debilitarse significativamente. Por ello muchos autores (ver p.ej. Olson, 2010) comparten que o bien la evolución está siendo en forma de "W" y las recuperaciones experimentadas vendrán seguidas de nuevas caídas o, en el peor de los casos, que no existe recuperación real sino un periodo largo de recorrido por el fondo (asemejándose a una "L").

En las economías desarrolladas, especialmente en Europa, se ha estancado antes de alcanzar los niveles previos al estallido de la crisis. En el conjunto de la región UNECE la mitad de las economías tenían en 2012 un PIB inferior al que tenían en 2008. Para el conjunto de la región UNECE el PIB es un $14 \%$ inferior al que se esperaba con la tendencias existentes antes del 2008. Esta cifra es más del doble que la del resto de las economías del mundo (que ronda el 6\%). (UNECE/FAO 2012). En la ZE la producción industrial es un $12 \%$ inferior a la existente a mediados de 2008 . (UNECE/FAO, 2012). Como resultado, los niveles de desempleo permanecen altos en Europa y América del Norte y todo apunta a que permanecerán así por algún tiempo. Con estos niveles de desempleo, la reducción de ingresos familiares, un excesivo número de viviendas sin vender y un sector financiero aún noqueado por la crisis de 2008-2009 y los problemas asociados a la deuda soberana de algunos países, nada parece apuntar a la existencia de cimientos suficientemente sólidos para una recuperación.

No obstante en regiones en desarrollo se mantiene el crecimiento económico, aunque con grandes variaciones entre países. Desde 2010, los países en desarrollo ha venido experimentando una razonable recuperación económica. Esta recuperación es más evidente en países de economías en transición ricos en recursos energéticos como Rusia y otros países de la antigua URSS. Asia ha sido el motor del crecimiento mundial en los últimos años, pero, aparentemente, su crecimiento se ha ralentizado en 2013 añadiendo tensión al cóctel económico mundial.

Casi seis años después del comienzo de la Gran Recesión, las predicciones sobre la evolu- ción económica global siguen dominadas por la incertidumbre derivada de la escasez y duración de las medidas de estímulo fiscal en economías avanzadas, el inesperado frenazo del crecimiento Chino, o la crisis de la zona euro.

\section{LA INDUSTRIA FORESTAL MUNDIAL EN LA RECESIÓN}

La Gran Recesión ha afectado negativamente todos los sectores industriales y comerciales, con una pérdida importante de capital humano, condicionando a trabajar con stocks mínimos y bajo pedidos confirmados, obligando a las empresas a refugiarse en sus actividades principales y con volúmenes reducidos por descenso de la demanda, causando un retroceso en la inversión en tecnología, y castigando con una escasa -si no nulaflexibilidad financiera. (OLSON, 2010).

Viendo las consecuencias generales sobre la industria en general es fácil suponer por qué la industria forestal fue especialmente golpeada por la crisis económica. Además, el hecho de que, en muchos países, el sector sea muy dependiente de la construcción de viviendas, agravó, obviamente, la situación. En los países desarrollados la contracción en la producción y la disminución del consumo se volvieron trascendentes en casi todos los segmentos de la industria de productos madereros desde 2008. Se han sucedido, desde entonces, los cierres de industrias, el desempleo, la caída de la producción, la ralentización del comercio y la pérdida de confianza de los consumidores. Este impacto alcanzó una de sus máximas expresiones en EE.UU. donde el comercio de madera de coníferas cayó en un $50 \%$ respecto a la de 2005; el de madera de frondosas un $35 \%$, los tableros estructurales un $37 \%$, los productos de madera mecanizados para construcción un 30\%. (TAYLOR, 2009). Pese a no estar directamente vinculadas con la construcción, las industrias de pasta y papel sufrieron también los efectos adversos debidos a la recesión económica general y el descenso del consumo (Tabla 1).

La industria de la madera sufrió un triple impacto. Al ya descrito brusco descenso de la demanda de sus productos, le acompañaron los problemas de financiación y acceso al crédito provocados por la crisis financiera, y las, a pri- 


\begin{tabular}{|c|c|c|c|c|c|c|c|c|c|c|}
\hline \multicolumn{2}{|l|}{ Unidades (miles) } & \multirow[t]{2}{*}{2008} & \multirow[t]{2}{*}{2009} & \multirow[t]{2}{*}{2010} & \multirow[t]{2}{*}{2011} & \multirow[t]{2}{*}{2012} & \multicolumn{2}{|c|}{$\begin{array}{c}\text { Cambio } \\
\text { 2008-2012 } \\
\text { Volumen \% }\end{array}$} & \multicolumn{2}{|c|}{$\begin{array}{c}\text { Cambio } \\
\text { 2011-2012 } \\
\text { Volumen \% }\end{array}$} \\
\hline \multicolumn{6}{|l|}{ Europa } & & & & & \\
\hline Madera en rollo industrial & $\mathrm{m}^{3}$ & 391.129 & 341.965 & 385.749 & 386.432 & 382.399 & -8.730 & $\mid-2,2$ & -4.033 & $-1,0$ \\
\hline Madera Aserrada & $\mathrm{m}^{3}$ & 101.682 & 92.204 & 101.865 & 101.475 & 98.575 & -3.107 & $-3,1$ & -2.900 & $-2,9$ \\
\hline Tableros de Madera & $\mathrm{m}^{3}$ & 69.850 & 61.545 & 66.572 & 67.444 & 66.910 & -2.940 & $-4,2$ & -534 & $-0,8$ \\
\hline Papel y Cartón & $\mathrm{t}$ & 97.417 & 87.802 & 94.371 & 92.871 & 89.119 & -8.298 & $-8,5$ & -3.752 & $-4,0$ \\
\hline \multicolumn{11}{|l|}{ CIS } \\
\hline Madera en rollo industrial & $\mathrm{m}^{3}$ & 112.176 & 100.916 & 126.271 & 172.122 & 171.894 & 59.718 & 53,2 & -228 & $-0,1$ \\
\hline Madera Aserrada & $\mathrm{m}^{3}$ & 18.170 & 17.412 & 17.449 & 17.764 & 18.289 & 119 & 0,7 & 525 & 3,0 \\
\hline Tableros de Madera & $\mathrm{m}^{3}$ & 14.367 & 10.531 & 12.661 & 15.432 & 15.814 & 1.447 & 10,1 & 382 & 2,5 \\
\hline Papel y Cartón & $\mathrm{t}$ & 9.120 & 8.466 & 9.363 & 9.594 & 9.248 & 128 & 1,4 & -346 & $-3,6$ \\
\hline \multicolumn{11}{|l|}{ Norte America } \\
\hline Madera en rollo industrial & $\mathrm{m}^{3}$ & 465.990 & 398.388 & 414.253 & 433.392 & 444.936 & -21.054 & \begin{tabular}{|l|}
$-4,5$ \\
\end{tabular} & 11.544 & 2,7 \\
\hline Madera Aserrada & $\mathrm{m}^{3}$ & 110.386 & 83.456 & 87.308 & 87.039 & 93.176 & -17.210 & $-15,6$ & 6.137 & 7,1 \\
\hline Tableros de Madera & $\mathrm{m}^{3}$ & 52.030 & 46.726 & 47.261 & 45.649 & \begin{tabular}{l|l}
46.399 \\
\end{tabular} & -5.631 & $-10,8$ & 750 & 1,6 \\
\hline Papel y Cartón & $\mathrm{t}$ & 89.743 & 78.443 & 83.244 & 79.721 & 78.001 & -11.742 & $-13,1$ & -1.720 & $-2,2$ \\
\hline \multicolumn{11}{|l|}{ Total UNECE } \\
\hline Madera en rollo industrial & $\mathrm{m}^{3}$ & 969.294 & 841.269 & 926.273 & 991.9451 & .025 .420 & 56.126 & 5,8 & 33.475 & 3,4 \\
\hline Madera Aserrada & $\mathrm{m}^{3}$ & 230.237 & 193.072 & 206.623 & 206.278 & 210.039 & -20.198 & $-8,8$ & 3.761 & 1,8 \\
\hline Tableros de Madera & $\mathrm{m}^{3}$ & 136.247 & 118.802 & 126.493 & 128.525 & 129.123 & -7.124 & $-5,2$ & 598 & 0,5 \\
\hline Papel y Cartón & $\mathrm{t}$ & 196.280 & 174.710 & 186.977 & 182.185 & 176.368 & -19.912 & $-10,1$ & -5.817 & $-3,2$ \\
\hline \multicolumn{11}{|l|}{ ESPAÑA } \\
\hline Madera Aserrada & $\mathrm{m}^{3}$ & 5.347 & 3.469 & 3.212 & 3.070 & 3.541 & -1.806 & 33,8 & 471 & 15,3 \\
\hline Tableros de Madera & $\mathrm{m}^{3}$ & 2.952 & 1.919 & 2.183 & 1.826 & 1.749 & -1.203 & $-40,7$ & -77 & $-4,2$ \\
\hline Papel y Cartón & $\mathrm{t}$ & 7.551 & 6.742 & 7.766 & 6.544 & 6.158 & -1.392 & $-18,4$ & -385 & $-5,9$ \\
\hline
\end{tabular}

Tabla 1. Evolución 2008-2012 del consumo aparente de productos forestales en la Región UNECE. Fuente: Adaptado de FAO 2013

mera vista paradójicas, dificultades de suministro de materia prima (Figura 5).

En una primera aproximación parecería que la caída de consumo de madera en rollo provocada por el descenso de la demanda de productos forestales y la subsiguiente caída de la producción de la industria de transformación, debería provocar un exceso de oferta de madera en rollo y un descenso automático de los precios y en igual o mayor cuantía. Esto no es tan sencillo por varios motivos. En primer lugar, la madera en pie no es un producto perecedero por lo que ante un empeoramiento repentino del mercado, la decisión del productor forestal es normalmente aplazar temporalmente la venta antes que asumir un descenso importante del precio. Esto es, obviamente, mucho más importante en el caso de pro- pietarios privados o compañías de inversión forestal y menos en el caso de superficies forestales gestionadas por empresas transformadoras. En segundo lugar, una parte importante de los costes de suministro de madera en parque de fábrica viene impuesta por los costes de aprovechamiento y transporte. Estos costes no dependen de los precios de los productos finales y por tanto no pueden ser fácilmente modificados. Más al contrario, en un escenario de aumento de los costes del petróleo tenderán a aumentar.

Otro factor que colaboró a dificultar el suministro de madera en los primeros años de recesión y que venía anteriormente condicionando mayores costes de aprovisionamiento, fue la necesidad de consumo de una determinada cantidad de madera certificada. En 2013 la superfi- 


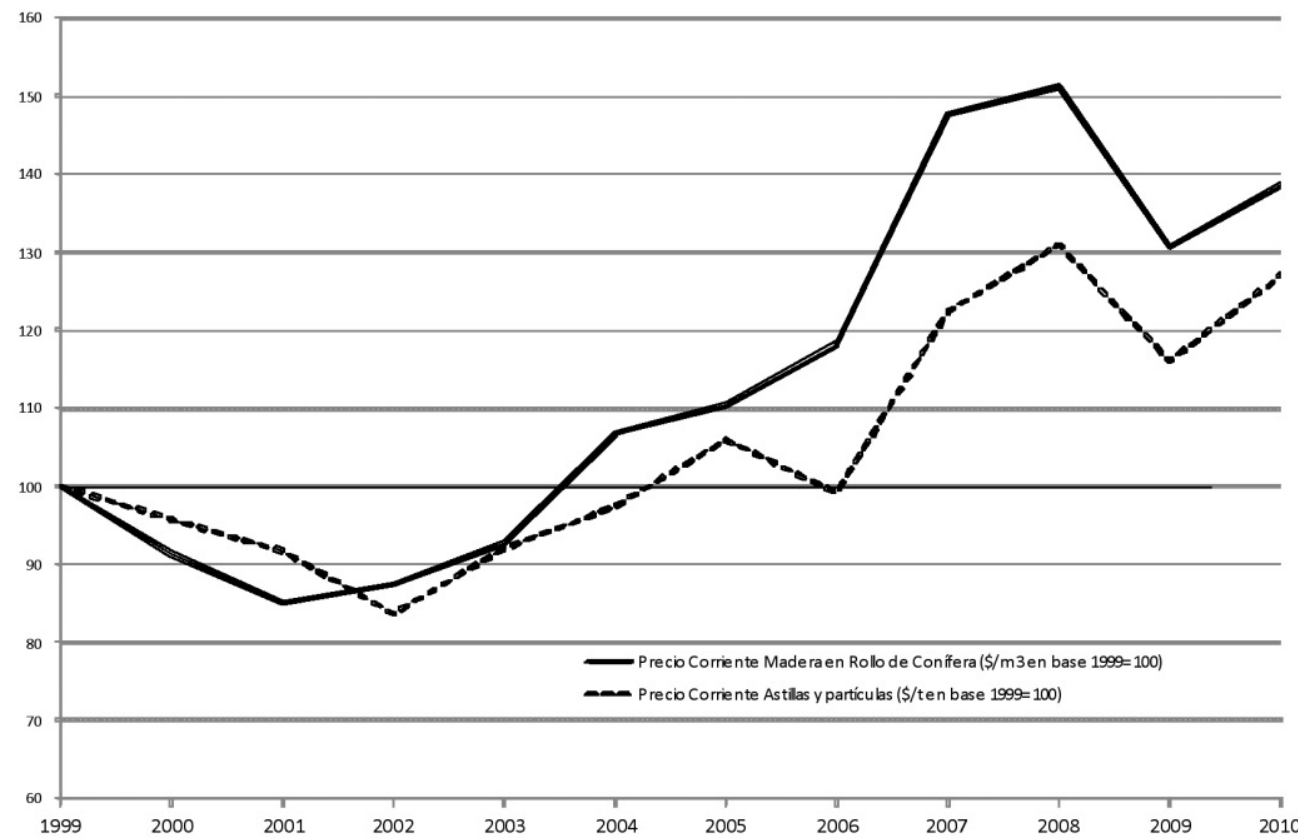

Figura 5. Evolución de precios medios corrientes de exportación (base 1999) de algunas materias primas (elaborado a partir de FAOSTAT 2012)

cie forestal certificada alcanzó el $10 \%$ de la superficie forestal total (UNECE/FAO 2013). No obstante el reparto de esta superficie no era homogéneo. En 2012 el $92 \%$ de la superficie forestal certificada se encontraba en el Hemisferio Norte. Se estima que la producción "potencial" de madera en rollo certificada procedente de esta superficie sería de 469 millones de $\mathrm{m}^{3}$, o lo que es lo mismo, un $27 \%$ de la producción global de madera en rollo. Durante la recesión el descenso de demanda de productos forestales afectó en menor medida a los productos certificados que a los no certificados. UNECE/FAO (2011) afirmaba que no hay evidencia de que los productos forestales certificados puedan alcanzar generalizadamente mayores precios en el mercado. Sin embargo, en un entorno de bajada generalizada de precios, la certificación ha actuado como elemento diferenciador y clave para el mantenimiento de ciertos clientes. Además, los canales de distribución en grandes superficies de bricolaje o mobiliario sufrieron menor caída de ventas que el comercio tradicional, mientras que simultáneamente, por presiones de grupos ambientales y sus propia actividades de promoción de imagen, aumentaron sus requerimientos de madera certificada, transmitiendo la obligación a sus proveedores.

Otro factor que colaboró a dificultar el aprovisionamiento incluso en etapas recesivas fue la competencia con usos energéticos para la madera. El aumento de los costes de la energía, y la implantación de políticas de incentivo a la bioenergía ha venido aumentando los consumos de madera para generación de energía térmica y eléctrica en países desarrollados. A modo de indicador baste citar que en 2012 los países de la UE27 aumentaron un $45,6 \%$ el consumo de energía a partir de biomasa forestal y madera respecto 2002 o que desde 2009 la importación de pellets de madera industriales creció en 2,5 millones de toneladas. (UNECE/FAO 2013).

Complicaciones adicionales para el suministro de madera por parte de la industria europea vinieron ocasionados por el cambio de tendencia en la relación euro/dólar y episodios de devaluación relativa del primero lo que encareció las importaciones desde terceros países. 


\section{LA INDUSTRIA FORESTAL ESPAÑOLA EN LA RECESIÓN}

La industria forestal y de la madera en España, salvo algunas excepciones en los sectores del papel y el tablero, es un sector muy fragmentado. Además existe una patente rotura de la cadena de la madera entre primeras y segundas transformaciones, al no ser significativas los enlaces entre la industria de la madera y la de fabricación de muebles (IGE 2013).

España es un país tradicionalmente deficitario en madera. Desde el comienzo de los registros estadísticos el consumo aparente ha estado siempre sensiblemente por encima de la oferta interna de productos. De hecho, en el año 2000 el porcentaje de autoabastecimiento apenas alcanzaba un $55 \%$ de consumo aparente. (VIGNOTE et al., 2010). Si bien en el decenio de bonanza económica previa a 2008, el mercado español de madera creció, la cuota de producto español ha ido disminuyendo. Este descenso de la endémica tasa de cobertura deficitaria y de la tasa de penetración de productos en los mercados internos, implica según GonZÁLEZ (2011) que no se logra competir con el producto importado, fabricado en condiciones ventajosas.

$\mathrm{Si}$ analizamos su evolución en el tiempo, se observa que el complejo productivo que va desde la selvicultura, la primera y segunda transformación de la madera (CNAE, 2009 2, 16, 17 y 31) ha ido perdiendo peso en la economía independientemente del ámbito en el que se estudie. Así en Galicia en el año 2000 representaba el 3,0\% del Valor Añadido Bruto (VAB) total de la economía y en el 2009, el 1,8\%. En España representaba el 2,1\% del VAB en el año 2000 y el $1,3 \%$ en 2009 y en Europa representaba el $2,1 \%$ del VAB en el año 2000 y el $1,6 \%$ en 2009. Esta tendencia está relacionada con la pérdida general de peso de las actividades industriales en favor de las del sector servicios.

No obstante IGE (2013) para el caso de Galicia establece que la pérdida de peso de la cadena forestal-madera dentro de la economía gallega es el resultado de una evolución diferente de estas ramas frente al resto de la economía. Así, la economía gallega creció en el período 20002004 la una tasa media anual del $6,9 \%$ a precios corrientes, mientras que este complejo productivo lo hacía a una tasa del 0,4\%. En el período 20052007 (etapa expansiva de la economía) se reducen las diferencias entre ambas tasas, pero, con todo, la cadena forestal- madera crece 3,7 puntos por debajo del total de la economía. Por último, en el período 2008-2010 (fase recesiva de la economía) las ramas de actividad que integran este complejo productivo decrecen a una tasa media del $-7,9 \%$ frente al $-1,4 \%$ del total de la economía. Esto pone de manifiesto que, durante los años de crecimiento económico, el VAB de la cadena crecía a un ritmo menor que el resto de ramas de la economía, mientras que, durante los años de crisis, la caída en el complejo es mayor.

La Gran Recesión global, supuso para España la explosión de otros problemas especialmente el final de la llamada "burbuja inmobiliaria", la crisis bancaria de 2012, el aumento del desempleo y fuertes descensos de consumo. La gran exposición de la industria de la madera al sector de la construcción, en el que una parte relevante de las empresas había basado su crecimiento en el decenio anterior, supuso un impacto inmediato de la crisis.

En España, desde 2008, el consumo aparente de madera aserrada descendió un $33,8 \%$, el de tableros un $40,7 \%$ y el de Papel y Cartón un $18,4 \%$ (Tabla 1). Pese a que este enorme descenso del consumo provocado por la recesión, eleva el porcentaje de autoabastecimiento a cifras inéditas -según VIGNOTE et al. (2010) en 2009 se alcanzó el $90 \%$ - la fabricación de productos se desmorona. Consecuentemente los Índices de Producción Industrial para los CNAE2009 16 y 31 (Industria de la Madera y Fabricación de Muebles, respectivamente) se situaron en valores nunca alcanzados desde el comienzo de la serie estadística del INE (Figuras 6,7 y 8).

De estos datos se concluye que la recesión ha causado sobre la industria forestal y de la madera en España un impacto similar al que ha provocado en la industria española en general. No obstante, haciendo un análisis por subsectores resulta evidente la especial incidencia que está teniendo la crisis en el subsector de fabricación de muebles, que ha reducido su actividad en más del $60 \%$, duplicando la reducción media del sector industrial de España.

GONZÁlEZ (2011) cuantifica un descenso anual de unos 100.000 empleos en la industria de la madera entre 2008 y 2009 aunque advier- 


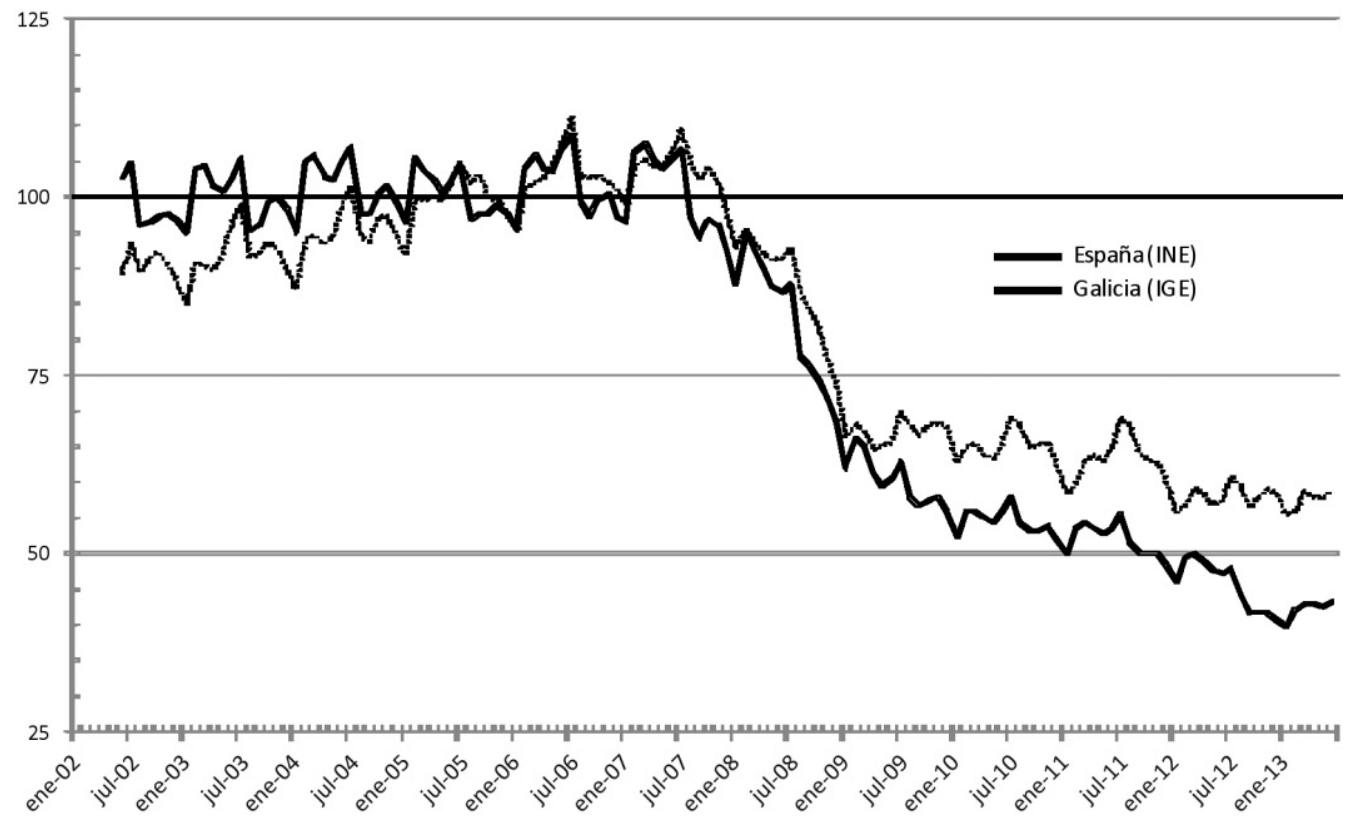

Figura 6. Evolución de la media móvil semestral del Índice de Producción Industrial para la Industria de la Madera en España y Galicia (CNAE 16)

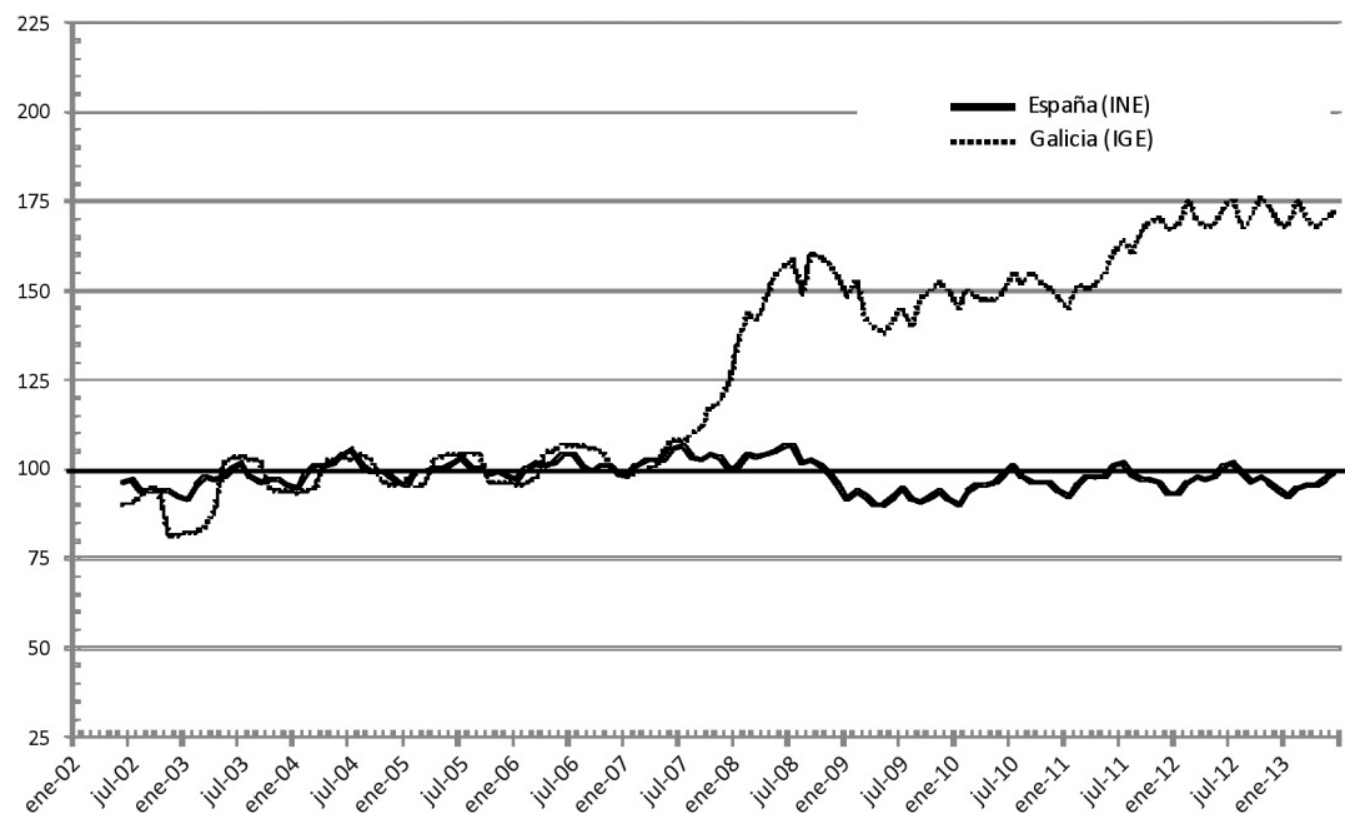

Figura 7. Evolución de la media móvil semestral del Índice de Producción Industrial para la Industria del papel en España y Galicia (CNAE 17) 


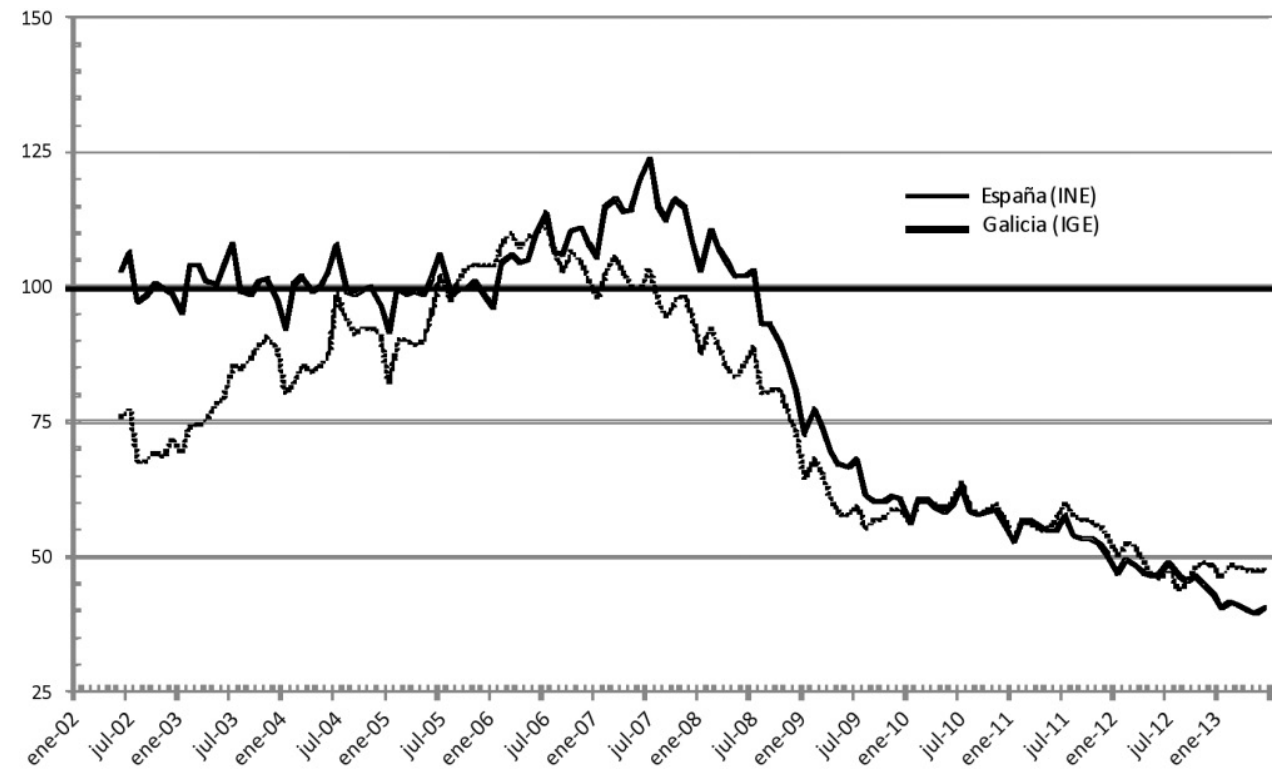

Figura 8. Evolución de la media móvil semestral del Índice de Producción Industrial para el sector de fabricación de muebles en España y Galicia (CNAE 31)

te, a partir de datos sectoriales y la Encuesta de Población Activa, una ralentización de la destrucción de empleo en 2010 y un leve incremento subida del mismo en 2011.

Pese a ello, por el enorme impacto que el desempleo y las políticas de contención salarial y gasto público está teniendo en el consumo, existe poca esperanza de cambios positivos basados en la demanda interna a corto plazo. Ante esto la búsqueda de mercados en el exterior no encuentra un panorama demasiado alentador. Salvo el caso de subsectores donde antes de la recesión primaba la exportación (pasta de papel, tablero) no existe capacidad exportadora que pudiera paliar el descenso de la demanda interna. Según GoNZÁLEZ (2011) esto es debido a la falta de posicionamiento del producto/marca en el mercado internacional. Además, se ha producido una importante deslocalización de la producción probablemente irreversible.

\section{EL SECTOR GLOBAL QUE VENDRÁ DESPUÉS DE LA TORMENTA}

En los países desarrollados, la contribución de las masas forestales al desarrollo económico, aun- que su aportación al PIB sea relativamente modesta, es significativa sobre todo por su potencial de desarrollo en un contexto de "Economía Verde" (UNECE/FAO, 2013b). Pese a los esfuerzos que el sector forestal ha estado realizando en comunicación, la sociedad no es aún plenamente consciente del papel que bosques y áreas forestales tienen en la producción de bienes y servicios que necesitan en su vida diaria, o cual es su contribución en el empleo o desarrollo económico, especialmente de zonas rurales. Por el contrario la opinión pública percibe, en general, a la industria forestal, como un sector anticuado de filosofía meramente extractiva. Sin embargo, pese a su enorme variabilidad entre subsectores y países, los hechos muestran un sector industrial que ha sido capaz de adaptarse a los cambios tecnológicos, sociales y económicos ocurridos durante su larga historia de trabajo basado en un recurso renovable incomparable como lo es la madera.

Autores como HeIFETZ et al. (2009) apuntan que la recesión no debe interpretarse como una simple "mala racha" que en algún momento terminará. Aunque en algunos mercados o zonas geográfica la fase de emergencia parezca haber terminado, lo que vendrá después es una fase de 
adaptación a la nueva realidad muy diferente del entorno en el que estaban antes de 2008.

Anteriores periodos de crisis económica han evidenciado que algunas empresas pueden encontrar oportunidades entrando en mercados donde los competidores se han debilitado o incluso pueden incorporar medio de producción o incluso talento que de otra manera no estarían disponibles (McKINSEY, 2009). Aquellas empresas que por precaución, modelo de negocio, intuición o meramente por azar actuaron antes de 2008 mejorando su exposición a la recesión, pese a que hayan sufrido un periodo adverso, están mejor posicionadas para sobrevivir que sus congéneres. No obstante es imprescindible que además deban llevar a cabo estrategias que apuntalen esta posición, aumenten su resiliencia. Estrategias de supervivencia como reducciones de capacidad productiva, descenso de mano de obra, reducción de gasto, reducción de plazos de entrega (y, de ser posible, cobro), y gestión conservadora de la liquidez, pueden suponer una ganancia de tiempo para las empresas pero no le proporcionarán ventajas competitivas adicionales en el futuro.

Los indicios de que el futuro vendrá en un entorno económico dinámico, incierto y frecuentemente turbulento, deberían obligar a las empresas y sectores económicos a adoptar estrategias de flexibilidad y agilidad ante movimientos competitivos. Los términos contenidos en la última frase no son en general los que el lector aplicaría generosamente a la industria forestal, y menos cuanto más al principio de la cadena de transformación de la madera nos encontremos. La dependencia de la materia prima y con esta de la gestión de recursos forestales (ya sea del mismo área geográfica o de áreas lejanas), la gran necesidad de capital, la característica de commodity de buena parte de los productos elaborados salvo en fases muy avanzadas y específicas de la transformación, han venido obligando a unidades productivas muy grandes, muy poco flexibles y con poca diferenciación de producto.

Por su intensidad, y por la propia naturaleza de la industria forestal, la Gran Recesión, incluso cuando haya finalizado, continuará condicionando los mercados y las empresas de productos basados en el bosque durante bastante tiempo. Aunque el futuro sea incierto, las exigencias por parte de consumidores y agentes sociales se mantendrán y las presiones económicas serán más pronunciadas. Las empresas deberán emplear mayores esfuerzos en gestionar su comunicación corporativa tanto interna como externa.

La madera y las fibras vegetales han encontrado su papel relevante en todos los periodos de la historia. Según PöYRY (2012) no hay nada que indique de de aquí a 2050 los productos basados en madera y fibra no tengan una importancia decisiva. Muchos gobiernos y organismos internacionales creen que la industria forestal posee un gran potencial para promover una "economía verde" a través del uso de la madera, la bioenergía y nuevos biomateriales (UNECE/FAO, 2013b) Por ello, en general, es difícil pensar en políticas futuras que puedan desincentivar el uso de productos forestales (JONSSON 2012).

Sin embargo el éxito de los mismos vendrá condicionado por la percepciones de los consumidores y en la capacidad del sector de promover su uso. La reciclabilidad de la inmensa mayoría de los productos forestales debería beneficiarse de una eventual mayor concienciación ambiental por parte de los consumidores y de políticas de gestión de residuos más exigentes. No obstante, teniendo en cuenta los escenarios debilidad económica de los consumidores futuros respecto a los del inmediato pasado, el comportamiento ambiental favorable no podrá por si mismo decantar al consumidor frente a opciones más competitivas en precio.

Además de la competencia con otros productos, la competencia intrasectorial aumentará y los países más desarrollados seguirán perdiendo cuota de mercado debido a sus mayores costes de producción y menores retornos de la inversión comparados con países en desarrollo que, además, dispongan de recursos forestales competitivos. Esto será especialmente patente en aquellos productos de menor valor añadido y commoditties, donde el factor crítico es el coste de suministro y producción, pero también será extensivo en otros segmentos. Por ello, se extenderán las estrategias de diferenciación de producto, fidelización de cadenas de suministro y de clientes, y estrategias de origen incluso bordeando los límites de los tratados de libre comercio. La importancia de la Calidad, hasta ahora la estrategia diferenciadora más empleada, parece que pueda ir decreciendo con los años ya que similares tecnologías de 
producción acabarán por estar implantadas en cualquier país productor.

Los sistemas de certificación forestal seguirán jugando un papel cualitativamente relevante. Pero se prevé que estén sometidos a una mayor competencia mutua y sobre todo de otras iniciativas a las que deberán adaptarse como políticas de lucha contra el cambio climático, aprovechamientos ilegales, bioenergía y biomateriales, comercio justo de productos forestales, etc. El desarrollo de iniciativas de "Green Building" en Europa, EE.UU. y Asia se espera que continúe y tenga un impacto significativo en los productos de madera y la selección de materiales de construcción que cumplan determinados criterios de contenido reciclado, biodegradable y de fuentes locales. Además los sistemas de certificación serán revisados y adaptados en el contexto de programas gubernamentales de lucha contra el comercio de madera procedente de fuentes conflictivas, como la Lacey Act en EE.UU. y la $E U$ Timber Regulation. Los beneficios de la certificación y otros mecanismos de apoyo a la gestión forestal sostenible basados en el mercado deberán adaptarse en términos técnicos y económicos para no penalizar, como hasta ahora, a los pequeños propietarios privados no industriales y permitirles un acceso justo al mercado.

En términos generales, durante las últimas cuatro décadas, la innovación tecnológica en la industria forestal ha sido modesta en comparación con buena parte del resto de sectores de la economía (VocEs, 2010). Es esperable, en aquellas áreas candidatas a liderar la economía basada en el bosque, que prime un enfoque basado en la innovación y en la mejora tecnológica, que obviamente, necesitará de inversiones tanto en I+D como en dotar a la mano de obra de conocimientos y medios tecnológicos. Por otra parte, la aproximación tradicional para la mejora de beneficios en la industria de transformación de madera ha venido consistiendo simplemente en aumentar el rendimiento sobre la madera en rollo transformada o aumentar la cantidad transformada. Hoy en día, la carestía de la madera, ha hecho que, tan importante como la gestión de las cantidades sea la gestión del valor de la madera producida, el grado de transformación, los subproductos generados por la misma y los canales de comercialización de los productos.
El incremento de consumo futuro de biomasa forestal para la generación de energía será una compleja función dependiente de los compromisos políticos para el fomento de energías renovables, los costes de producción, el apoyo financiero público, los precios generales de la energía y los otros combustibles, las preferencias sociales y de los consumidores, entre otros. Las políticas públicas de establecimiento de objetivos energéticos y creación de instrumentos de apoyos financieros han ayudado en los últimos años al crecimiento de la producción de energía a partir de biomasa forestal y madera. Por ejemplo, en 2012 los países de la UE27 aumentaron un $45,6 \%$ el consumo de energía a partir de biomasa forestal y madera respecto 2002. (UNECE/ FAO 2013). La reducción de los presupuestos de las administraciones públicas en los años venideros puede reducir significativamente el acceso de promotores a la recepción de ayudas directas, beneficios fiscales o, simplemente, financiación. Aunque todos los escenarios dan por hecho un crecimiento del mercado de los biocombustibles de origen forestal re reconoce generalizadamente que existen grandes incertidumbre sobre la disponibilidad de biomasa y sus precios (STELTE, 2012).

En el campo de los biomateriales, especialmente el de los bioplásticos, se han venido sucediendo un gran número de innovaciones de especial aplicación en los segmentos de la higiene, salud y embalaje/packaging. Estos productos innovadores están encontrando interesantes nichos en las industrias electrónicas y de automoción. Además, productos de "madera técnica" y elementos de madera mecanizados han proporcionado importantes avances para el uso innovador de madera en construcción con propiedades muy mejoradas desde el punto de vista estructural, de durabilidad, estético y ambiental. La implantación de estas soluciones constructivas se está llevando a cabo con desigual ritmo, dependiendo de las tradiciones locales de construcción, la presencia de industria forestal proveedora y el alcance de las inversiones y apoyos públicos. Por otra parte las nuevas biorrefinerías están siendo capaces de poner en el mercado valiosos y útiles productos químicos, algunos de ellos capaces de sustituir otros tradicionales derivados del petróleo. Como es obvio, las incertidumbres sobre la 
disponibilidad en cantidad, claridad y precio de madera, biomasa forestal y subproductos de la cadena de transformación tradicional de la madera, es un aspecto que, si cabe aquí con mayor incertidumbre planea sobre el futuro.

Como se ha mostrado en anteriores párrafos, la disponibilidad de madera en términos económicos y adecuados a las exigencias sociales y ambientales futuras es un aspecto clave que se enfrenta a importantes incertidumbres.

A escala global, la tierra con fines productivos va a ser un recurso escaso en el futuro en todos los sentidos. El aumento de áreas urbanizadas, roturadas, o degradadas y los efectos del cambio climático disminuirán en amplias zonas geográficas la disponibilidad de tierra para fines productivos. Complementariamente, la reserva, con objetivos conservadores de otras zonas, imposibilitará, dificultará o cuando menos descenderá la productividad real de otras áreas forestales. En un futuro con aumento de demanda de madera, será necesario garantizar, en los terrenos disponibles, una mejora de la productividad a partir de gestión más intensa, mejora genética, etc.

Son numerosos los estudios prospectivos del suministro futuro de madera y su adecuación a la potencial demanda. Entre ellos es destacable el llamado EFSOS II "European Forest Sector Outlook Study II', promovido por la UNECE/FAO (2011b) y cuyo ámbito es la UE27, y los países del Este y Sureste de Europa. (JONSSON, 2012; MANTAU, in press). Dicho estudio asume que entre 2010 y 2030 la demanda de madera en Europa aumente, en el caso más optimista, un $21 \%$ (201,7 millones de $\mathrm{m}^{3}$ de madera en rollo equivalente) y un 47\% (453,2 millones de $\mathrm{m}^{3}$ en rollo equivalente) en el caso de que se cumpliesen los objetivos de producción bioenergética. Incluso éste último escenario, parte de supuestos muy favorables de: eficiencia energética; importantes incrementos de otras energías renovables; modestos crecimientos del empleo de madera para elaboración de materiales tradicionales y nuevos biomateriales; y manteniendo el mismo área forestal. EFSOS II establece que, por tanto, para incrementar el suministro de madera en las tasas previstas podría hacer necesario emplear superficies de producción adicionales mediante el establecimiento de cultivos de crecimiento rápido sobre terrenos agrícolas. En este sentido, concluye que obtener 100 millones de $\mathrm{m}^{3}$ adicionales obligaría, con productividades medias, a utilizar unos 5 millones de hectáreas en este sentido.

\section{SIN CARTAS DE NAVEGACIÓN TODOS LOS MARES SON DESFAVORABLES}

La industria forestal española no ha sido estudiada de una forma integral con tanto rigor como otros sectores económicos o incluso otros ámbitos del mundo agrario. Esto es especialmente patente en lo relativo a su estructura productiva y relación con los mercados. Según (DÍAZ BALTEIRO et $a l ., 2008$ ) las razones que pueden justificar este hecho probablemente tengan que ver tanto con peculiaridades internas como con la dificultad de obtener estadísticas precisas de diversas variables relacionadas, propias del ámbito forestal. Es indudable que además de la complejidad del sector, la dejadez de las administraciones responsables y la incapacidad de la compleja estructura asociativa del sector de tomar la iniciativa son cooperadores necesarios. La inexistencia de mínimas fuentes estadísticas actualizadas sobre caracterizaciones subsectoriales, consumos de madera, posibilidades de suministro en términos económicos de madera por parte de los montes españoles, impiden que en estos momentos se puedan elaborar diagnósticos adecuados y ayudar en la toma de decisiones tanto en el ámbito empresarial como en el ámbito público.

En el símil de la tormenta que encabeza este artículo, la situación sería comparable a darse cuenta en medio del temporal que se desconoce las características del barco y de que se carece de cartas náuticas o faros que pudieran ayudaran a fijar un rumbo más seguro.

Complementariamente a esta reflexión se ha de tener en mente que, en muchos casos se habla del impacto de la recesión sobre el sector como si una parte importante de los efectos que está teniendo no hubieran existido previamente a la misma, enmascarados en la bonanza económica, en la disponibilidad de crédito o en recursos públicos inadecuadamente empleados. A veces al marino también se le olvida que el mar era tan profundo en la calma como lo es ahora en la tempestad. 


\section{BIBLIOGRAFÍA}

Díaz Balteiro, L.; Herruzo, A.; Prieto, A.; Romero, C.; GonzÁlez, J.; Calvo, X.; MarTÍNEZ, M. Y Voces, R.; 2008. Caracterización de la industria forestal en España. Fundación BBVA. Madrid.

Elsby, M.W.L; Hobijn, B. \& Sahin, A; 2010. The Labor Market in the Great Recession. Brookings Papers on Economic Activity, Economic Studies Program 41(1): 1-69.

FAO; 2011. State of the World's Forests, 2011. Food and Agriculture Organization of the United Nations). Rome.

FAO; 2013; FAOSTAT Database on Agriculture. Food and Agriculture Organization of the United Nations. Rome, Italy. http://faostat.fao.org

GONZÁLEZ, V.; 2011; El sector de la madera en España. Factores de competitividad y perspectivas del sector. Estrategias de distribución. Congreso Emclaves 2011. Confemadera.

Heifetz, R., Grashow, A. \& Linsky, M.; 2009. Leadership in a permanent crisis. Harvard Business Review 87(7-8): 62-69. http://www.unece.org/fileadmin/DAM/timber/docs/efsos/03-sept/dp-30.pdf

IGE; 2013. Análise da cadea forestal-madeira; Instituto Galego de Estatística. Ed. Xunta de Galicia, Consellería de Facenda. Colección: Área de estatísticas económicas. Santiago de Compostela.

Jonsson, R.; 2012. Econometric Modelling and Projections of Wood Products Demand, Supply and Trade in Europe - A contribution to EFSOS II. Geneva Timber and Forest Discussion paper UNECE. ECE/TIM/DP/59. Geneva.

Krugman, P.; 2006. Economic Storm Signals. Columna de opinión en New York Times 1 Diciembre 2006. http://www.nytimes.com/2006/12/01/opinion/01krugman.html

Mantau, U.; (in press); The Method of the Wood Resource Balance - A contribution to EFSOS II. Geneva Timber and Forest Discussion paper UNECE. ECE/TIM/DP/60. Geneva.

MCKINSEY; 2009. Navigating the downturn. McKinsey of Finance 30 (Winter 2009): 1-40.

NBER; 2010; Business Cycle Dating Committee Report Sept.20th 2010. NBER National
Bureau of Economic Research. http://www.nber.org/cycles/sept2010.html

Olson, E.G.; 2010. Supply chain opportunity in an uncertain economic recovery. Supply Chain Management: An International Journal 15(6): 488-492.

PÖYRY; 2012; Future from fibre From forest to finished product. Technical report ed by Pöyry for the Forest Solutions Group of the World Business Council for Sustainable Development (WBCSD) in cooperation with WWF International. http://www.wbcsd.org/Pages/EDocument/EDocumentDetails.aspx?ID=15244

Stelte, W.; 2012; Global Market for Wood Pellets and Price Development. Danish Technological Institute. In cooperation with IEA - Bioenergy Task 40.

TAYLOR, R.; 2009. Crisis en la industria y en los mercados de productos madereros: perspectivas desde América del Norte. Unasylva 233: 13-22.

UNECE/FAO; 2011; Forest Products Annual Market Review, 2010-2011. UNECE/FAO. Geneve.

UNECE/FAO; 2011b. The European forest sector outlook study II, 2010-2030; EFSOS II. UNECE and FAO. Geneve.

UNECE/FAO; 2012. Forest Products Annual Market Review, 2011-2012. UNECE and FAO. Geneve.

UNECE/FAO; 2013. Forest Products Annual Market Review, 2012-2013 (Advance). UNECE and FAO. Geneve.

UNECE/FAO; 2013b. Forest and Economic Development: a Driver for the Green Economy in the ECE Region. ECE/TIM/SP/31 United Nations Publications.

Vignote, S; AMBRosio, Y; MARTíNEZ, I. Y GONZÁLEZ, V.; 2010. Análisis de la evolución del mercado de la madera en España y perspectivas de desarrollo. Boletín AITIM 268: 66-73.

Vlosky, R. \& PANWAR, R.; 2012. Competing in the global economic recession. In: IUFRO Conference Division 5 Forest Products. Final program, proceedings and abstracts book: 41-45. Estoril.

VocES, R; 2010. Análisis de la innovación y la sostenibilidad en la industria forestal. Tesis Doctoral. Universidad Politécnica de Madrid. Madrid. 